\title{
Numerical simulation of atomization process of nickel- based alloy powders prepared by vacuum induction melting gas atomization
}

\author{
Kuai-Kuai Guo ${ }^{1}$, Jin Chen ${ }^{2}$, Shuo Shang ${ }^{3}$, Chang-Sheng Liu ${ }^{4}$ \\ School of Material Science and Engineering, Northeastern University, Shenyang, China \\ ${ }^{4}$ Corresponding author \\ E-mail: ${ }^{1}$ neukuai@163.com, ${ }^{2} 18202421691 @ 163 . c o m,{ }^{3}$ sshang@mail.neu.edu.cn, ${ }^{4}$ lxnnd777@163.com \\ Received 11 March 2020; accepted 24 March 2020 \\ DOI https://doi.org/10.21595/vp.2020.21386 \\ Check for updates \\ Copyright $(C 2020$ Kuai-Kuai Guo, et al. This is an open access article distributed under the Creative Commons Attribution License, \\ which permits unrestricted use, distribution, and reproduction in any medium, provided the original work is properly cited.
}

\begin{abstract}
ANSYS Fluent 19.0 software is used to numerically simulate atomization process in the production of nickel-based alloy powders by VIGA. The gas flow field in the atomizing chamber and atomization process of the metal melt is studied. Atomizing air flow is sprayed through the nozzle to form supersonic gas flow. The results show that the recirculation zone is inverted cone, and the length of recirculation zone is $12.28 \mathrm{~mm}$. Under the stagnation point, the velocity direction of the air flow is downward, and the velocity increases gradually (the maximum is $457.43 \mathrm{~m} / \mathrm{s}$ ). In gas-liquid two-phase state, the length of recirculation zone is about $16 \mathrm{~mm}$. The higher the turbulence in the recirculation zone and turbulent layer, the more intense the interaction between primary and secondary atomization.
\end{abstract}

Keywords: CFD, VIGA gas atomization, primary and secondary atomization, recirculation zone.

\section{Introduction}

Gas atomization is a preparation method which uses high kinetic energy gas (atomizing medium) to impact the high-temperature melt of metal or alloy into small droplets, and then rapidly solidify into solid powder $[1,2]$. Vacuum induction melting gas atomization (VIGA) is a widely used method to prepare metal powder. Generally, it is divided into two main stages [3]: the primary atomization, also known as one-time crushing, refers to the cylindrical flow of high-temperature molten alloy flowing out of the delivery tube, which interacts with the high-speed gas flow field, disperses and forms a liquid film, and then breaks into an initial droplet; the second atomization, also known as the second crushing, refers to the process that the initial droplet is entrained by the air flow to the turbulent layer. That is, the process of secondary crushing and further crushing into smaller droplets.

The breaking process of molten metal is a coupling process of multiphase flow, including primary atomization and secondary atomization, which is difficult to be characterized by conventional experimental means $[4,5]$. There are two traditional methods to study and solve the problem of fluid flow: one is pure theoretical analytical fluid mechanics method, the other is experimental fluid mechanics method. With the development and progress of computer technology, numerical simulation has become a very convenient and effective method. In engineering application, especially in solving complex flow problems, numerical simulation method has obvious advantages and is paid more and more attention.

In this paper, the interaction mechanism of solid, liquid and gas in the atomization process of superalloy is studied, and the influencing factors and control of powder characteristics in the process of melt breaking, spheroidizing and solidification are studied. The numerical calculation method of CFD, a commercial software ANSYS fluent, is used to simulate the preparation process of VIGA nickel base alloy powder. 


\section{Experimental procedure}

In this paper, CFD method ANSYS fluent 19.0 commercial software is used to simulate the preparation process of atomized nickel base alloy powder by vacuum induction melting, including the primary atomization process and the secondary atomization process of high temperature alloy melt. Considering that in the actual atomization process, the flow field takes the nozzle axis as the center line and presents an axisymmetric distribution, the three-dimensional structure of the actual center symmetry is simplified as a two-dimensional axisymmetric structure. The simplified geometry model of VIGA aerosol equipment is shown in Fig. 1. As the calculation area is two-dimensional axisymmetric geometry, in order to reduce the calculation amount, half model is selected as the calculation area, and gambit software is used to mesh the flow field area.

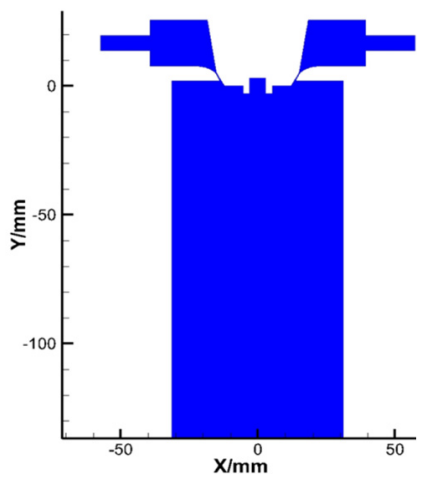

Fig. 1. Simplified geometric model of the gas atomizer

In the simulation process, for the primary atomization process, the physical model is VOF multiphase flow model and large eddy model. For the secondary atomization process, the physical model is DPM discrete phase model. For the crushing process, the TAB model is used.

\section{Results and discussion}

Fig. 2 is the velocity cloud diagram and velocity vector diagram of the recirculation zone at the bottom of the diversion pipe. Under the bottom of the draft tube, there is an inverted cone-shaped recirculation zone with the opposite direction of the air flow velocity and the inlet air flow. The boundary between the recirculation zone and the high-speed jet is the sonic line. In the circumfluence area, because of the speed difference between the inside and outside air flow, the air flow guide pipe in the center of the circumfluence area moves and rotates radially outwards when approaching the bottom of the melt delivery tube. When the flow contacts the sonic boundary, it is pushed inward and flows downstream. There is a stagnation point at the end of the recirculation zone, the gas velocity is 0 , and the Mach disk (positive shock) is about $25 \mathrm{~mm}$ below the stagnation point.

Fig. 3 shows the velocity curve on the center line of the atomizer. It can be seen that the velocity of the gas in the delivery tube is 0 , the lower part of the tube is far away from the direction of the guide pipe, the direction of the air velocity in the recirculation zone is positive, the velocity value first increases (the maximum value can reach $240.39 \mathrm{~m} / \mathrm{s}$ ), then decreases (the velocity value is 0 ), and the point with the velocity of 0 is called the stagnation point. Between the bottom of the diversion pipe and the stagnation point is the range of the recirculation zone. According to Fig. 3 , the length of the recirculation zone is $12.28 \mathrm{~mm}$. below the stagnation point, the velocity direction of the air flow is downward, and the velocity increases gradually (the maximum is $457.43 \mathrm{~m} / \mathrm{s}$ ). When it reaches the Mach disk position, the velocity value decreases gradually due to the blocking effect of the Mach disk; the influence area of the Mach disk is limited, and the velocity in the downstream direction increases gradually; thus, the velocity is gradually stable. 


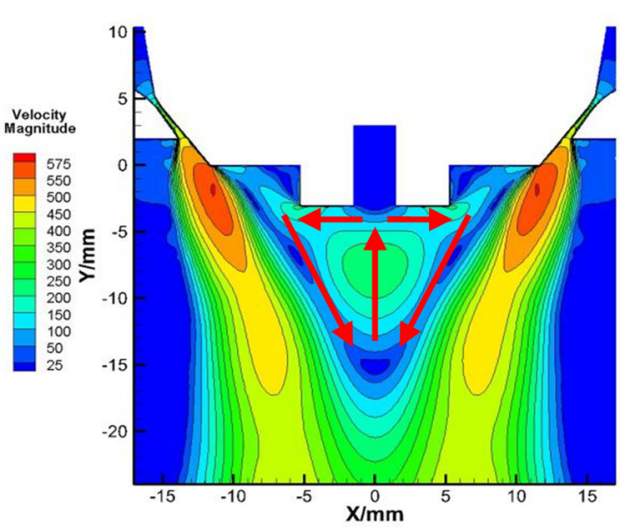

a)

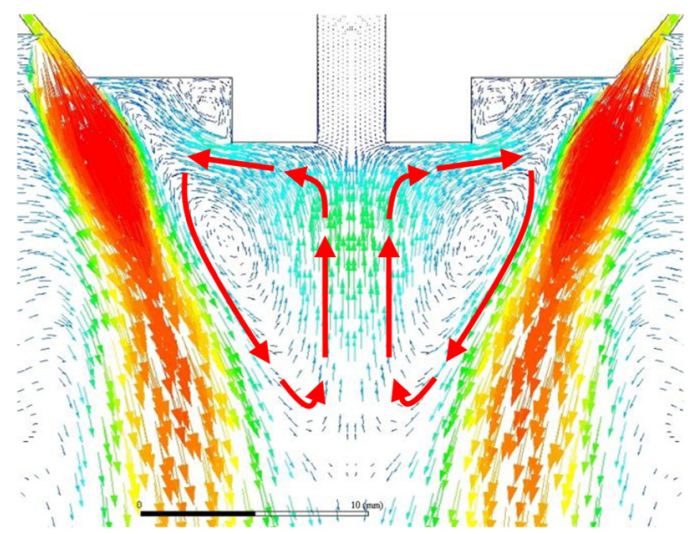

b)

Fig. 2. Gas velocity flow field of recirculation zone

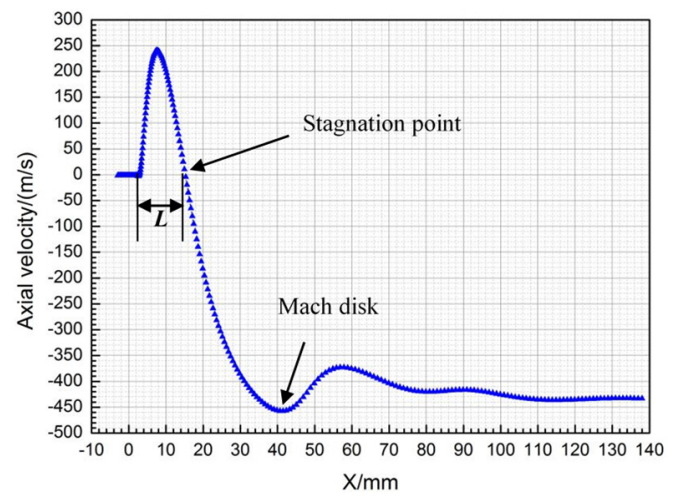

Fig. 3. Velocity curve along central axis of gas flow field

Velocity field of gas-liquid two-phase flow field is shown in Fig. 4. The continuous flow of molten liquid column in the guide tube is blocked by the air flow upward in the recirculation zone, "divided" into long strips, which flow and disperse radially outwards in the form of supporting umbrellas, thus gradually separating from the main molten metal flow continuously flowing out of the delivery tube. As can be seen from Fig. 4(b), the length of the recirculation zone is about $16 \mathrm{~mm}$, which is longer than that of the single-phase flow field. In addition, due to the existence of many small-scale vortices, the speed of the air flow changes more diversified. Only within the length of $16 \mathrm{~mm}$ of the recirculation zone, the air flow speed has experienced three changes of increasing first and then decreasing.

It can be seen from Fig. 4(c) that the recirculation zone in equilibrium is disturbed by the high-temperature melt entering the atomization area, and many low-speed vortices are formed in the recirculation zone. This is because the kinetic energy of the air flow is converted into the surface energy of the metal melt, and the formation of the metal liquid film and the droplet hinders the normal flow direction of the air flow. The turbulent layer between the flow area and the supersonic area is also affected by these melts, forming more small and disordered eddy current areas, and the local air flow speed is reduced, but the eddy current speed in the recirculation zone is much higher, forming a very disordered turbulent layer, which is the main area for the secondary atomization, effectively breaking the large droplets of metal melts into smaller droplets. The higher the degree of turbulence in the recirculation zone and turbulent layer, the more intense the interaction between the primary and secondary atomization, the more fully the metal film and large droplets are broken, and the larger the disturbance range of gas outflow is. 


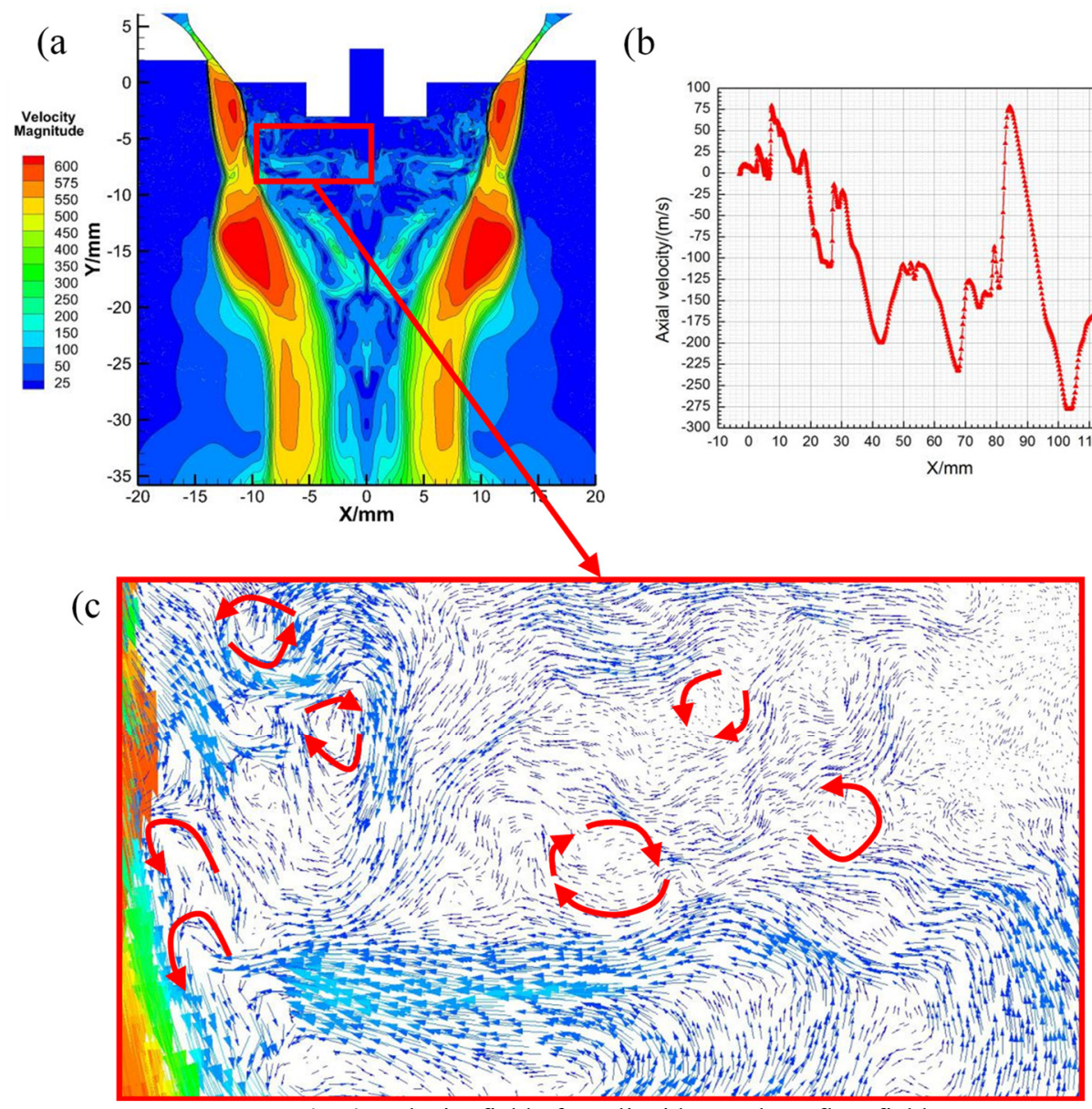

Fig. 4. Velocity field of gas-liquid two-phase flow field

\section{Conclusions}

Atomizing air flow is sprayed through the nozzle to form supersonic gas flow. The recirculation zone is inverted cone, and the length of recirculation zone is $12.28 \mathrm{~mm}$. Below the stagnation point, the velocity direction of the air flow is downward, and the velocity increases gradually (the maximum is $457.43 \mathrm{~m} / \mathrm{s}$ ). In gas-liquid two-phase state, the length of recirculation zone is about $16 \mathrm{~mm}$. The higher the turbulence in the recirculation zone and turbulent layer, the more intense the interaction between primary and secondary atomization.

\section{Acknowledgements}

This work was financially supported by National Key R\&D Program of China (2017YFB0305801), Joint-Fund of NSFC-Liaoning (U1508213) and Natural Science Foundation of China (51771051).

\section{References}

[1] Dawes J., Bowerman R., Trepleton R. Introduction to the additive manufacturing powder metallurgy supply chain. Johnson Matthey Technology Review, Vol. 59, Issue 3, 2015, p. 243-256. 
[2] Heidloff A. J., Rieken J. R., Anderson I. E., et al. Advanced gas atomization processing for Ti and Ti alloy powder manufacturing. Journal of the Minerals, Metals and Materials Society, Vol. 62, Issue 5, 2010, p. 35-41.

[3] Philippe V., Zuzio D., Estivalezes J. L., et al. Numerical simulation of primary and secondary atomization. Comptes Rendus Mécanique, Vol. 341, Issue 1-2, 2013, p. 15-25.

[4] Xia M., Wang P., Zhang X. H., et al. Computational fluid dynamic investigation of the primary and secondary atomization of the free-fall atomizer in electrode induction melting gas atomization process. Acta Physica Sinica, Vol. 67, Issue 17, 2018, p. 170201.

[5] Li X. G., Fritsching U. Process modeling pressure-swirl-gas-atomization for metal powder production. Journal of Materials Processing Technology, Vol. 239, 2016, p. 1-17. 